\title{
Pharmacological advances in the management of osteoporosis
}

\author{
Noel P Somasundaram ${ }^{1}$, M S A Cooray ${ }^{2}$ \\ Sri Lanka Journal of Diabetes, Endocrinology and Metabolism 2012; 2: 92-100
}

\begin{abstract}
Osteoporosis is a worldwide health problem with a high prevalence. Agents for the treatment of osteoporosis are classified as antiresorptives, anabolic agents and drugs with combined anabolic and anti-resorptive actions.

Although many drugs with proven efficacy are available for the treatment of osteoporosis their effectiveness has been limited by side-effects, concurrent comorbidities, and inadequate long-term compliance. Additionally, conventional antiresorptives such as aminobisphosphonates profoundly suppress bone resorption and formation which might contribute to the pathogenesis of osteonecrosis of the jaw.

Various novel antiresorptive agents are in development. This overview aims to discuss in brief some of the most promising novel treatments which include: bazedoxifene a new selective estrogen receptor modulator, denosumab, PTH rP (parathyroid hormone related protein), odanacatib and other bone anabolic agents such antibodies against sclerostin and dickkopf-1.

Denosumab, a fully human monoclonal antibody to receptor activator of nuclear factor kappa B ligand (RANKL) an anti-resorptive agent with a low side effect profile has been proven efficacious. Bazedoxifene has also proven its efficacy. Odanacatib, an inhibitor of cathepsin $\mathrm{K}$, which is an osteoclast enzyme required for resorption of bone matrix is under assessment as an anti-resorptive agent with no antianabolic effects and is showing promising results.

Anabolic agents act by stimulating formation of new bone. Novel agents in development include: antibodies to sclerostin and dickkopf-1, proteins that target molecules involved in Wnt signaling, a pathway that regulates gene transcription of proteins that are important for osteoblast function; an antagonist to the calcium-sensing receptor; and an activin receptor fusion protein, which functions as an activin antagonist and has shown promise as an anabolic agent in early human trials.
\end{abstract}

\section{Introduction}

Osteoporosis, the most common metabolic bone disease, is characterized by low bone mineral density (BMD) and microarchitectural deterioration of bone tissue consequently leading to an increase in the susceptibility to fragility fractures. The problem of osteoporosis has become so important due to the ageing population (1). Currently, every third postmenopausal woman and every fifth man older than 50 years suffer from osteoporosis. In a cross-sectional survey of a group of 1642 communitydwelling postmenopausal women in seven provinces in Sri Lanka using peripheral dual-energy X-ray absorptiometry revealed that 736 women (44.9\%) were likely to have osteoporosis (2). A similar study reported 5.8\% prevalence of osteoporosis among men older than 50 years in Sri Lanka (3).

Bone is a dynamic tissue, which has the ability to adapt its shape and size in response to mechanical loads through a phenomenon of remodeling. This process allows the skeleton to maintain mechanical integrity through constant osteoclastic resorption of damaged bone followed by osteoblast-mediated deposition and mineralization of new matrix. Bone strength, ie, resistance to fracture, depends not only on bone mass, but also on its spatial distribution and the intrinsic properties of the materials that constitute the bone (1). The main aim of treatment of osteoporosis is to reduce the bone fracture risk as far as possible. Although it is not possible to eliminate the entire risk for fractures, currently available medications can substantially reduce that risk. Even small increases in bone mass can substantially reduce the incidence of fracture.

Anti-osteoporosis drugs can be classified on the basis of their action on bone remodeling. Antiresorptive drugs decrease bone resorption and reduce fractures by preserving skeletal microarchitecture. Anabolic drugs on the other hand, reduce fractures by enhancing remodeling. In addition to increasing BMD, they appear to repair bone microarchitecture and improve bone geometry. There are also drugs that decouple the two processes, inhibiting bone resorption and stimulating bone formation (Table 1).

${ }^{1}$ Consultant Endocrinologist, National Hospital of Sri Lanka, Colombo, ${ }^{2}$ Senior Registrar in Endocrinology, National Hospital of Sri Lanka, Colombo. 
Table 1. Osteoporosis therapies in use

\begin{tabular}{llccc}
\hline Drug class & Drugs & $\begin{array}{c}\text { Action on bone } \\
\text { remodeling }\end{array}$ & $\begin{array}{c}\text { Action on bone } \\
\text { mineral density }\end{array}$ & $\begin{array}{c}\text { Action on vertebral } \\
\text { and nonvertebral } \\
\text { fracture risk }\end{array}$ \\
\hline Antiresorptive agents & Aminobisphosphonates & $\downarrow \downarrow$ & $\uparrow$ & $\mathrm{V} \downarrow ; \mathrm{NV} \downarrow$ \\
& Raloxifene & $\downarrow$ & $\uparrow$ & $\mathrm{V} \downarrow$ \\
Uncoupling agent & Strontium ranelate & $\downarrow \mathrm{NC}, \uparrow \mathrm{OB}$ & $\uparrow$ & $\mathrm{V} \downarrow ; \mathrm{NV} \downarrow$ \\
Anabolic agents & PTH 1-84 & $\uparrow$ & $\uparrow \uparrow$ & $\mathrm{V} \downarrow ; \mathrm{NV} \downarrow$ \\
& Teriparatide & $\uparrow$ & $\uparrow \uparrow$ & $\mathrm{V} \downarrow ; \mathrm{NV} \downarrow$ \\
& & $\uparrow$ & $\mathrm{V} \downarrow ; \mathrm{NV} \downarrow$ \\
\hline
\end{tabular}

Notes: $\uparrow$ increase; $\downarrow$ decrease; $\uparrow \uparrow$ or $\downarrow \downarrow$ strong increase or decrease, respectively. Adapted from International Journal of Clinical Rheumatology, June 2011, Vol. 6, No. 3, Pages 359-369.

Abbreviations: OB, osteoblast; OC, osteoclast; NV, nonvertebral; PTH, parathyroid hormone; V, vertebral.

\section{Important issues and limitations with regards to some of the currently used drugs}

Three different aminobisphosphonates (alendronate, risedronate, and zoledronate), teriparatide, strontium ranelate, and hormone replacement therapy have been shown to be effective against both vertebral and nonvertebral fractures. Evidence of efficacy against vertebral fractures is available for ibandronate (an aminobisphosphonate), raloxifene, and PTH 1-84 (1).

Unfortunately despite effective therapies, because of patients' unwillingness to take current therapies or inability to tolerate the therapies, many patients remain untreated.

\section{Bisphosphonates}

They are well established, widely used, first line treatment agents to treat osteoporosis, and they act via the inhibition of osteoclasts, leading to reduced bone turnover, increased bone mass, and improved mineralization (5). Bisphosphonates can be given orally or intravenously, and relatively inexpensive and used across a broad spectrum of osteoporosis types, including postmenopausal, male, and steroid-induced osteoporosis, as well as Paget's disease.

Recent evidence have raised some important issues

- In addition to inhibiting osteoclasts, bisphosphonates (specifically alendronate) may also promote osteoblast proliferation and maturation $(4,6)$.

- Alendronate and zoledronate by binding strongly to the bone matrix and becoming internalized within bone suppresses bone resorption continuously (even after treatment cessation) - this has lead to recent concerns of detrimental changes to bone quality and accumulation of damage (5).

\section{Strontium ranelate}

It is a bone-seeking agent capable of increasing bone formation and reducing bone resorption, thereby uncoupling and rebalancing bone turnover in favor of bone formation (7). Although trials have proven efficacy against vertebral and non-vertebral fractures over 5 years in women with postmenopausal osteoporosis no studies have been performed on strontium ranelate efficacy on men (8-9).

\section{Parathormone}

Both the full-length parathyroid hormone (PTH 1-84) and its N-terminal fragment, teriparatide (PTH 1-34) are bone-anabolic drugs targeting osteoblasts and are potent stimulators of bone formation that restore bone to an osteopenic skeleton when administered intermittently. They have the potential to increase bone mass and reduce fracture risk dramatically. Currently, the only anabolic agent approved by the FDA for the treatment of osteoporosis in the United States is teriparatide, a 1-34 amino acid fragment of human parathyroid hormone (PTH 1-34). In Europe, a full-length PTH (1-84) molecule also is approved for therapy.

A limitation of PTH is the need for daily subcutaneous administration. Therefore, alternative delivery systems, such as oral, transdermal, and intranasal, have been tested. A recent study comparing the transdermal daily administration of teriparatide verses subcutaneous administration demonstrated a good bioavailability and an increase in vertebral BMD comparable with subcutaneous teriparatide $(10,11)$. Although teriparatide has revolutionized the 
management of osteoporosis there have been some issues of concern (13-15):

- despite bone mass increase the new bone is less mineralized.

- the protective action of teriparatide on BMD vanishes with time in both genders (but not up to the baseline values) in contrast to bisphosphonates, the effect of which persists for many months after drug withdrawal

- concurrent therapy with antiresorptive therapy, particularly bisphosphonates, has to be avoided

- higher cost

- hypercalcemia - which is associated with increases in osteoclast-driven bone resorption.

- side-effects such as nausea, flushing and muscle cramping.

- the induction of osteosarcoma in a rat model of carcinogenicity (12).

Thus teriparatide therapy is not recommended for more than 2 years, and contraindicated in patients at increased risk of osteosarcoma (these include Paget disease of bone, open epiphyses, a history of irradiation involving the skeleton, or an unexplained elevation of alkaline phosphatase level of skeletal origin).

Although concurrent treatment is not recommended it has been demonstrated that sequential treatment with antiresorptives after teriparatide or PTH prevents accelerated osteoclastic resorption of the new bone tissue built during teriparatide therapy, increases mineralization, and rapidly lowers cortical porosity; leading to further increases in BMD (15).

\section{Estrogens and selective estrogen receptor modulators (SERM)}

These have effects not only on bone metabolism, but also on the breast, endometrium, and lipid metabolism. Proven effects of SERM on bone density and metabolic bone markers have been generally more modest than that seen with bisphosphonates. In the Multiple Outcomes of Raloxifene Evaluation (MORE) trial, although vertebral fractures were decreased by $37 \%$ after four years of raloxifene, a reduction in non-vertebral fractures was not seen (16). Adverse events such as increase in hot flushes and a 3-fold increase in venous thromboembolic events are associated with use of raloxifene.

\section{New therapies}

Management of osteoporosis is challenging and research continues to design a drug that is not only safe and cost-effective but also with the following characteristics.
- Reduce fracture risk without long-term deleterious effects on the skeleton or other side effects

- Effects of the drug cease with cessation of therapy

- Inhibit bone resorption without inhibiting bone formation

Anabolic agents can target signals increasing the osteoblastic cellular pool or the function of the mature cell. Because of their potential to increase bone mass, extensive research is being carried out in this area. Although emphasis is being placed in the development of new anabolic agents, new antiresorptive agents are being pursued with the hope to develop therapies with good efficacy, tolerability, and simplicity of administration. This overview aims to discuss some of the most promising novel treatments which include:

\section{Anti-resorptive therapies}

- Bazedoxifene

- Denosumab

- Odanacatib

\section{Bone anabolic agents}

- Antibodies against the proteins sclerostin and dickkopf-1.

- $\quad$ PTH rP (Parathyroid Hormone related protein)

\section{Bazedoxifene (BZA)}

Bazedoxifene is a novel third-generation selective estrogen receptor modulator, a molecule developed to act as estrogen receptor agonists in some tissues (eg. bone) and as estrogen receptor antagonists in others, such as breast and endometrium, in order to reduce the risk of breast and endometrial cancers that would be induced by hormone replacement therapy (17-19).

Two large Phase III clinical trials have shown that bazedoxifene increased BMD, decreased levels of bone turnover markers, and significantly reduced the risk of new vertebral fractures in postmenopausal women compared with placebo $(17,18)$. Although the incidence of nonvertebral fractures with bazedoxifene or raloxifene did not differ significantly from that with placebo, a post hoc analysis of a subgroup of women at higher fracture risk revealed that bazedoxifene significantly reduced the nonvertebral fracture risk relative to placebo and raloxifene. There was no evidence of endometrial and breast stimulation in studies. Bazedoxifene use is associated with an improvement in the lipid profile (reduction in the serum concentrations of total cholesterol and low-density lipoprotein cholesterol, with an increase in the serum level of high-density lipoprotein cho-lesterol). The incidence of vasodilatation (hot flushes), leg cramps, and venous thromboembolic events were significantly higher with bazedoxifene compared with placebo. 


\section{Tissue selective estrogen complex (TSEC)}

TSEC is a novel therapy, partnering a selective estrogen receptor modulator (SERM) with one or more estrogens. Based on the favorable effects of bazedoxifene on the breast and endometrium, pairing of bazedoxifene with conjugated estrogens is done for the treatment of menopausal symptoms and prevention of postmenopausal osteoporosis. The pairing of bazedoxifene with conjugated estrogens (CE) has been evaluated in the Selective estrogens, Menopause, And Response to Therapy (SMART) phase 3 trials and overall, BZA/CE has been shown to be effective in relieving menopausal symptoms and treating osteoporosis while maintaining endometrial and breast safety $(1,17)$.

Although bazedoxifene is a promising new therapy for patients with osteoporosis, further clinical investigations of long-term treatment with this selective estrogen receptor modulator are needed to evaluate the prevention of osteoporotic fractures, breast cancers, endometrial cancers, and cardiovascular events (17).

\section{Denosumab}

Denosumab a human monoclonal antibody to RANKL (receptor activator of KB ligand) is a promising new drug for the prevention and treatment of postmenopausal osteoporosis. It has been approved by the Food and Drug Administration (FDA) and European Medicines Agency for the treatment of :

- postmenopausal osteoporosis in women at high risk of fracture.

- bone loss associated with androgen deprivation therapy in men with prostate cancer.

It may be particularly useful in clinical practice for the treatment of patients who have failed or are intolerant to other available osteoporosis therapy, eg. patients with gastrointestinal contraindications, side effects with oral bisphosphonates, or malabsorption. Denosumab has also shown promising skeletal effects in the treatment of cancer and rheumatoid arthritis $(20,21)$. Denosumab $60 \mathrm{mg}$ is given subcutaneously every six months and is simple to administer and well tolerated.

\section{Mechanism of action}

The principal regulator of bone resorption is the RANKL/RANK/ osteoprotegerin pathway. RANKL is a transmembrane and soluble protein that is highly expressed by osteoblasts; its receptor, RANK, is located on the cell membrane of osteoclasts and preosteoclasts $(22,23)$. RANKL-RANK binding stimulates the formation, activity, and survival of osteoclasts, resulting in increased bone resorption.

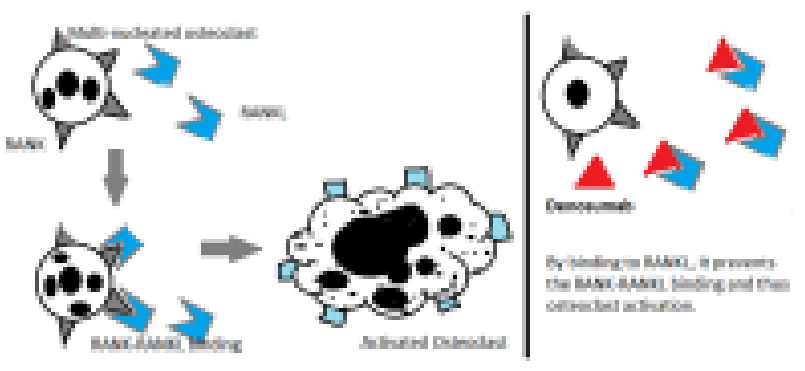

Figure. Denosumab - mechanism of inhibition of bone resorption.

Osteoprotegerin (OPG) is a naturally occurring, endogenous regulator "decoy receptor" of the RANKLRANK pathway. By binding to RANKL and preventing its interaction with RANK, OPG inhibits osteoclast formation, activity and survival thereby reducing bone resorption (24-26). Denosumab acts like osteoprotegerin, diminishing osteoclast activity by binding to RANKL with high affinity and specificity and blocking the interaction of RANKL with RANK (22).

\section{Denosumab in postmenopausal osteoporosis}

The FREEDOM (Fracture Reduction Evaluation of Denosumab in Osteoporosis Every 6 Months) is the largest and most important trial of four Phase III studies in testing denosumab in postmenopausal women. In this 3 year trial, 7868 women aged 60-90 years with a bone density $\mathrm{T}$ score $<-2.5$ in the lumbar spine or total hip were randomized to receive either denosumab $60 \mathrm{mg}$ or placebo by subcutaneous injection every six months for three years.(27). There was a statistically significant (68\%) relative risk reduction and an almost 5\% absolute risk reduction in the primary outcome which was a new vertebral fracture diagnosed by spine x-ray. There was a reduction in clinical vertebral fractures as well as nonvertebral fractures and bone mineral density increased relative to the placebo group by $9.2 \%$ (spine) and 6\% (total hip). Bone turnover markers reflecting both osteoclastic and osteoblastic activity were suppressed by denosumab compared with placebo $(20,28)$.

In DEFEND (Denosumab Fortifies Bone Density), a Phase III trial evaluating the efficacy and safety of denosumab in postmenopausal women with low bone mass (osteopenia), denosumab significantly increased $\mathrm{BMD}$ at lumbar spine compared with placebo at 24 months as well as at total hip, distal one-third radius, and total body (28). DECIDE (Determining Efficacy: Comparison of Initiating Denosumab Versus Alendronate) and STAND (Study of Transitioning from Alendronate to Denosumab) both 1-year Phase III trials in postmenopausal women with osteopenia/osteoporosis which compared denosumab 60 mg every 6 months with oral alendronate $70 \mathrm{mg}$ weekly revealed a significantly greater $\mathrm{BMD}$ increase with 
denosumab compared with alendronate at total hip and all other measured skeletal sites at 12 months (20,25,28). Discontinuing denosumab (at a dose of $210 \mathrm{mg}$ ) after 24 months resulted in a decrease in BMD in the following year comparable to the gains in BMD with 24 months of therapy. Denosumab has a declining residual effect over 1 year, a period called offset time.

\section{Denosumab in androgen deprivation therapy (ADT)}

Patients with prostate cancer on ADT have a higher risk for fracture, as high as $20 \%$ over five years, despite having a good overall survival outlook (29-32). Although effective, adherence to weekly or even monthly oral bisphosphonate has been unsatisfactory in these patients. Thus yearly intravenous zoledronic acid is the preferred agent. Denosumab is also attractive because it can be given subcutaneously at every other administration of ADT therapy, which is usually a gonadotropin hormonereleasing hormone analog provided every three months $(33,34)$.

\section{Important characteristics of denosumab therapy in comparison to bisphosphonates $(26,27,35)$ :}

1. Convenient biannual subcutaneous administration that could translate into improved long-term adherence.

2. Reversibility, because it targets RANKL and not incorporated into the bone mineral thus yielding a much shorter terminal half-life (26). Thus for the patient who has a side effect from therapy, denosumab will be no longer active six months after the last dose and will be an advantage. On the other hand, if patients are not receiving denosumab regularly, the patient's fracture risk might increase after the dose "wears off" (27).

3. Inhibits both osteoclastogenesis and osteoclast activation. These actions at different levels of osteoclast cell-biology could explain why bone biopsy samples from patients treated with denosumab have no osteoclasts in more than $50 \%$ of samples (20).

4. Lack of gastrointestinal side-effects.

5. May worsen hypocalcemia in patients with severe renal impairment (creatinine clearance $<30 \mathrm{~mL} / \mathrm{min}$ ) or on chronic haemodialysis (21).

6. Cleared by nonrenal metabolism - bisphosphonates are cleared by the kidney and contraindicated in patients with renal insufficiency. Denosumab may prove to be a safe drug in these patients, although studies that directly address this issue need to be done.

7. Serious skin infections, as well as infections of the abdomen, urinary tract, and ear, are more frequent in patients treated with denosumab. Epidermal and dermal adverse events (eg. dermatitis, eczema, and rashes) occurred at a significantly higher rate in the denosumab group compared to the placebo group in the FREEDOM trial (28).

8. The FREEDOM trial reported no cases of osteonecrosis of the jaw (ONJ) in either denosumab or placebo group (28). Although this may be a advantage over bisphosphonates patients should be monitored for consequences, including ONJ, atypical fractures, and delayed fracture healing as the effect of long-term treatment are unknown $(20,21)$.

\section{Odanacatib}

Odanacatib is currently the most advanced inhibitor of cathepsin $\mathrm{K}$ under clinical investigation. It is orally available and effective in a once-weekly $50 \mathrm{mg}$ dose (36). Cathepsins are lysosomal proteases that belong to the papain-like cysteine protease family. Out of the eleven different types that have been described (B, C, F, H, K, L, $\mathrm{O}, \mathrm{S}, \mathrm{V}, \mathrm{X}$, and $\mathrm{W}$ ), cathepsin $\mathrm{K}$ (being a key lysosomal enzyme of the mature activated osteoclast) is the most important with respect to bone remodeling, since it is a protease with intense collagenase activity. It is predominantly expressed in osteoclasts and degrades the two main types of collagen I and II. It is essential to dissolve calcic hydroxyapatite, the main mineral component of bone with respect to acid $\mathrm{pH}$ and is an attractive target for selective inhibition to reduce bone resorption (37-39).

\section{Important characteristics of cathepsin $\mathrm{K}$ inhibitors (36,} 40)

- Cathepsin K inhibition has shown a quality that is not present among other classes of antiresorptive agents in that it has resulted in greater suppression of bone resorption than bone formation, suggesting a dissociation between bone resorption and bone formation. This is due to preservation of osteoclast viability despite suppressed osteoclast function. These effects might allow osteoclast-to-osteoblast signalling that maintains bone formation while suppressing bone resorption. These uncoupling effects of odanacatib contrast with other antiresorptive drugs such as bisphosphonates and denosumab, which enhance osteoclast apoptosis.

- The onset and resolution for the antiresorptive effect of cathepsin $\mathrm{K}$ inhibitors is rapid. While a rapid resolution of effect might be preferable if there are concerns about side effects with prolonged exposure, it could also increase the risk of fracture shortly after discontinuation of treatment. This becomes particularly important in clinical practice where intended and unintended interruptions in therapy, including noncompliance, are very common. 
Phase I and II studies conducted in postmenopausal women showed odanacatib to be safe and well tolerated. After 5 years, women who received ODN 50 mg weekly showed significant BMD increases from baseline of 11.9\% at the lumbar spine, $9.8 \%$ at the femoral neck, $10.9 \%$ at the hip trochanter, and $8.5 \%$ at the total hip. A low antiresorptive activity with only slightly reduced bonespecific alkaline phosphatase was maintained through 5 years of treatment. In women who were switched from ODN to placebo after 2 years, bone turnover markers reverted to baseline levels after 12 months off medication. Adverse experiences in the ODN-treated group were not significantly different from the placebo group. However, there is yet no fracture data for ODN. Three Phase III studies with ODN are ongoing, two in postmenopausal women and one in men to assess its safety, efficacy and effects on fracture risk (41-43). The results of the BMD efficacy and safety of odanacatib in postmenopausal women trial, a clinical, randomized, double-blind trial with 16,716 patients, are expected in the latter half of 2012 (43).

\section{Parathyroid hormone-related protein (PTHrP)}

PTHrP is a naturally occurring polypeptide that is closely related to PTH and activates the same receptor as PTH (PTHR1). It is expressed in many tissues, but is found in high levels at the growth plate, in osteoblasts and within mammary tissues. It was initially identified as the circulating cause of hypercalcaemia of malignancy after being cloned from cancer cells. Although originally thought to be a potent stimulus of bone resorption and hence hypercalcaemia, experimental studies using intermittent PTHRP have shown that it has significantly similar anabolic properties to PTH1-34 (44-46).

Unlike PTH, which is a mixed anabolic and catabolic agent, PTHrP displays features suggesting that it may be a pure anabolic agent when intermittently administered. Studies have shown that short-term, high-dose treatment with PTHrP has yeilded encouraging results $(44,45)$. A study done by Horwitz MJ showed that intermittent PTHrP was administered safely and without serious adverse events in subjects receiving 500 and 625 microg/d for 3 wk. Subjects receiving $750 \mathrm{microg} / \mathrm{d}$ developed mild hypercalcemia (44). Bone turnover markers suggested that even at the highest doses, daily sc PTHrP may not activate bone resorption, i.e. may be purely anabolic. Interestingly, when hypercalcemia occurred, it may have resulted not from bone resorption but from activation of intestinal calcium absorption by 1,25 dihydroxyvitamin D. Thus, human PTHrP (1-36) is one of the promising drugs for treatment of osteoporosis. However, the long-term safety and fracture efficacy will only be determined by a large Phase III clinical trial (46).

\section{Calcilytic drugs}

These act as antagonists of the Calcium Sensing Receptor (CaSR) and mimic hypocalcaemia, thus evoking a short pulse of PTH secretion which translates into a bone-anabolic effect. The mechanisms responsible for the anabolic effect of the calcilytic agent in bone have not been reported, but it is likely that they are analogous to those of PTH. This hormone has mitogenic properties for cells of the osteoblastic lineage and increases the synthesis of IGF I by osteoblasts, resulting in an increase in bone collagen synthesis and bone formation (47). Calcilytics are given orally and obviate the need for injections, as opposed to PTH treatment. Currently newer calcilytic drugs with an improved pharmacological profile are being assessed. The most advanced compound of this class is MK-5442, which is currently in phase-2 trials for postmenopausal osteoporosis $(47,48)$.

\section{Sotatercept}

An antagonist to the calcium-sensing receptor and an activin receptor fusion protein, which functions as an activin antagonist, have shown promise as anabolic agents in early human trials. Sotatercept, a soluble activin receptor type 2A IgG-Fc fusion protein is being extensively evaluated in preclinical studies, in particular in models of cancer- and osteoporosis-related bone loss. In a phase I clinical trial in postmenopausal females, sotatercept increased hematocrit levels, and, in a phase II trial in patients with multiple myeloma, a trend toward improvement in osteolytic lesions as well as antitumor activity was observed (48).

\section{Inhibitors of Wnt antagonists and antibody therapies}

Wnts constitute a family of proteins important in cell differentiation. The $\mathrm{Wnt} / \beta$-catenin signaling pathway plays a critical role in osteoblastic the differentiation of mesenchymal cells toward mature osteoblasts and bone formation (49). Secreted Wnt antagonists include sclerostin and Dickkopf (Dkk-1).

Antisclerostin antibodies - Sclerostin, the product of the sost gene, inhibits osteoblastogenesis. Humanized monoclonal antibodies to sclerostin cause enhanced Wnt signaling. Phase I study in humans demonstrated that antisclerostin antibodies can increase BMD and biochemical markers of bone formation in humans. Phase II study is currently under way to assess the impact of a humanized antisclerostin antibody on BMD in postmenopausal women with low BMD (49).

Dkk-1 antibodies - Dkk-1 functions as an inhibitor of Wnt signaling and could be a potential therapeutic target for osteoporosis. Dkk-1 neutralization causes an increase 
in BMD, trabecular bone volume, osteoblast surface, and bone formation in rodents, suggesting that Dkk-1, like sclerostin, neutralization could be pursued as an anabolic approach in the treatment of osteoporosis, but no studies in humans have been reported (50).

The downside is that indiscriminate Wnt activation could result in unwanted side effects and possible tumorigenicity in nonskeletal tissues. Activating mutations of the Wnt signaling pathway are associated with colorectal cancer, hepatocellular carcinoma, and other malignancies (51).

\section{Other anabolic agents under investigation}

- Glucagon-like peptide 2 - prevents the nocturnal rise in bone resorption without affecting bone formation.

- Insulinlike growth factor 1 (IGF-1) - enhances the differentiated function of osteoblasts and bone formation (52).

\section{Conclusion}

Improved understanding of the molecular and cellular regulators and mediators of bone remodeling has lead to the identification of new targets for therapeutic intervention of osteoporosis. Denosumab, a fully human monoclonal antibody to Receptor Activator of Nuclear factor Kappa B ligand (RANKL), an anti-resorptive agent inhibits bone resorption and is approved for the treatment of women with postmenopausal osteoporosis at high risk of fractures. An inhibitor of cathepsin K, odanacatib, is in phase III clinical trials for the treatment of postmenopausal osteoporosis; it decreases bone resorption while seeming to suppress bone formation less than other antiresorptive agents (53).

Anabolic therapies are also fast developing. Investigational monoclonal antibodies to sclerostin have osteoanabolic properties with the potential to improve clinical outcomes in patients with osteoporosis (54).

PTHrP remains a potentially important future anabolic treatment for osteoporosis, particularly if this agent increases bone formation more than bone resorption.

Novel interventions that target newly recognized regulators of bone remodeling are promising agents for the treatment of osteoporosis and it is invariable that the number of available agents for the treatment of osteoporosis will dramatically increase in the coming years.

Multiple novel anti-osteoporotic compounds are in advanced clinical trials and are proving to be efficacious, convenient as well as with lesser side effect profile. However, long term clinical data will be needed before they are approved for clinical use.

\section{References}

1. Cavalli L, Brandi ML. Age- and gender-related macro and microarchitecture changes in bone structure and implications for treatment. Int J Clin Rheumatol 2011; 6(3): 359- 69.

2. Lekamwasam S, Wijayaratne L, Rodrigo M, Hewage U. Prevalence of osteoporosis among postmenopausal women in Sri Lanka: a cross-sectional community study. APLAR Journal of Rheumatology 2007; 10(3): 234-8.

3. Lekamwasam S, Wijayaratne L, Rodrigo M, Hewage U. Prevalence and determinants of osteoporosis among men aged 50 years or more in Sri Lanka: a community-based cross-sectional study. Arch Osteoporos 2009; 4(1-2): 79-84.

4. Gallacher SJ, Dixon T. Impact of treatments for postmenopausal osteoporosis (bisphosphonates, parathyroid hormone, strontium ranelate, and denosumab) on bone quality: a systematic review. Calcif Tissue Int 2010; 87(6): 469-84.

5. Reszka AA, Rodan GA. Bisphosphonates mechanism of action. Curr Osteoporos Rep 2003; 1(2): 45-52.

6. Xiong Y, Yang HJ, Feng J, Shi ZL, Wu LD. Effects of alendronate on the proliferation and osteogenic differentiation of MG-63 cells. J Int Med Res 2009; 37(2): 407-16.

7. Deeks ED, Dhillon S. Strontium ranelate: a review of its use in the treatment of postmenopausal osteoporosis. Drugs 2010; 70(6): 733-59.

8. Meunier PJ, Roux C, Seeman E, et al. The effects of strontium ranelate on the risk of vertebral fracture in women with postmenopausal osteoporosis. N Eng J Med 2004; 350(5): 459- 68.

9. Reginster JY, Seeman E, De Vernejoul MC, et al. Strontium ranelate reduces the risk of nonvertebral fractures in postmenopausal women with osteoporosis: Treatment of Peripheral Osteoporosis (TROPOS) study. J Clin Endocrinol Metab 2005; 90(5): 2816-22.

10. Daddona PE, Matriano JA, Mandema J, Maa YF. Parathyroid hormone (1-34)-coated microneedle patch system: clinical pharmacokinetics and pharmacodynamics for treatment of osteoporosis. Pharm Res 2011; 28(1): 159-65.

11. Cosman F et al. Effect of transdermal teriparatide administration on bone mineral density in postmenopausal women. J Clin Endocrinol Metab 2010; 95(1): 151-8.

12. Vahle JL, et al. Skeletal changes in rats given daily subcutaneous injections of recombinant human parathyroid hormone (1-34) for two years and relevance to human safety. Toxicol Pathol 2002; 30: 312-21.

13. Bone HG, Hosking D, Devogelaer JP, et al. Ten years' experience with alendronate for osteoporosis in 
postmenopausal women. $N$ Engl J Med 2004; 350(12): 1189-99.

14. Finkelstein JS, Wyland JJ, Leder BZ, et al. Effects of teriparatide retreatment in osteoporotic men and women. $J$ Clin Endocrinol Metab 2009; 94(7): 2495-501.

15. Eastell R, Nickelsen T, Marin F, et al. Sequential treatment of severe postmenopausal osteoporosis after teriparatide: final results of the randomized, controlled European study of Forsteo (EUROFORS). J Bone Miner Res 2009; 24(4): 726-36.

16. Kagan R. The tissue selective estrogen complex: a novel approach to the treatment of menopausal symptoms. $J$ Womens Health 2012. [Epub ahead of print]

17. Silverman SL, Christiansen C, Genant HK, et al. Efficacy of bazedoxifene in reducing new vertebral fracture risk in postmenopausal women with osteoporosis: results from a 3-year, randomized, placebo- and active-controlled clinical trial. J Bone Miner Res 2008; 23(12): 1923-34.

18. Miller PD, Chines AA, Christiansen C, et al. Effects of bazedoxifene on BMD and bone turnover in postmenopausal women: 2-yr results of a randomized, double-blind, placebo-, and active-controlled study. J Bone Miner Res 2008; 23(4): 525-35.

19. Kawate H, Takayanagi R. Efficacy and safety of bazedoxifene for postmenopausal osteoporosis. Clin Interv Aging 2011; 6: 151-60.

20. Reid I, Miller P, Brown J, et al, on behalf of the Denosumab Phase 3 Bone Histology Study Group. Effects of denosumab on bone histomorphometry: the FREEDOM and STAND studies. J Bone Miner Res 2010; 25: 2256-65.

21. Lewiecki EM. Treatment of osteoporosis with denosumab. Maturitas 2010; 66(2): 182-186.

22. Hsu H, Lacey DL, Dunstan CR, et al. Tumor necrosis factor receptor family member RANK mediates osteoclast differentiation and activation induced by osteoprotegerin ligand. Proc Natl Acad Sci USA 1999; 96(7): 3540-5.

23. Lacey DL, Timms E, Tan HL, et al. Osteoprotegerin ligand is a cytokine that regulates osteoclast differentiation and activation. Cell 1998; 93(2): 165-76.

24. Simonet WS, Lacey DL, Dunstan CR, et al. Osteoprotegerin: a novel secreted protein involved in the regulation of bone density. Cell 1997; 89(2): 309-19.

25. Baron R, Ferrari S, Russell RG. Denosumab and bisphosphonates: different mechanisms of action and effects. Bone 2011; 48(4): 677-92.

26. Adler RA, Gill RS. Clinical utility of denosumab for treatment of bone loss in men and women. Clin Interv Aging 2011; 6: 119-124.

27. Cummings SR, San Martin J, McClung MR, et al. Denosumab for prevention of fractures in postmenopausal women with osteoporosis. $N$ Engl J Med 2009; 361(8): 756-65.

28. Brown JP, Prince RL, Deal C, et al. Comparison of the effect of denosumab and alendronate on bone mineral density and biochemical markers of bone turnover in postmenopausal women with low bone mass: a randomized, blinded, phase 3 trial. J Bone Miner Res 2009; 14: 1-34.

29. Shahinian VB, Kuo YF, Freeman JL, Goodwin JS. Risk of fracture after androgen deprivation for prostate cancer. $N$ Engl J Med 2005; 352(2): 154-64.

30. Lu-Yao GL, Albertsen PC, Moore DF, et al. Survival following primary androgen deprivation therapy among men with localized prostate cancer. JAMA 2008; 300(2): 173-81.

31. Adler RA. Management of osteoporosis in men on androgen deprivation therapy. Maturitas 2011; 68(2): 143-7.

32. Smith MR. Androgen deprivation therapy for prostate cancer: new concepts and concerns. Curr Opin Endocrinol Diabetes Obes 2007; 14(3): 247-54.

33. Smith MR. Androgen deprivation therapy and risk for diabetes and cardiovascular disease in prostate cancer survivors. Curr Urol Rep 2008; 9(3): 197-202.

34. Bone HG, Bolognese MA, Yuen CK, et al. Effects of denosumab on bone mineral density and bone turnover in postmenopausal women. J Clin Endocrinol Metab 2008; 93(6): 2149-57.

35. Rachner TD, Khosla S, Hofbauer LC. Osteoporosis: now and the future. Lancet 2011; 9: 377(9773): 1276-87.

36. Castrillón JP, Pinacho F, Luis D, Menendez M, Laita AL. Odanacatib, a new drug for the treatment of osteoporosis: review of the results in postmenopausal women. $J$ Osteoporos 2010: 1-5.

37. Yasuda Y, Kaleta J, Brömme D. The role of cathepsins in osteoporosis and arthritis: rationale for the design of new therapeutics. Adv Drug Deliv Rev 2005; 57: 973-93.

38. Vasiljeva O, Reinheckel T, Peters C, Turk D, Turk V, Turk B. Emerging roles of cysteine cathepsins in disease and their potential as drug targets. Curr Pharm Des 2007; 13: 387-403.

39. Stoch SA, Wagner JA. Cathepsin K inhibitors: a novel target for osteoporosis therapy. Clin Pharmacol Ther 2008; 83: 172-6.

40. Boonen S, Rosenberg E, Claessens F, Vanderschueren D, Papapoulos S. Inhibition of cathepsin K for treatment of osteoporosis. Curr Osteoporos Rep 2012; 10(1): 73-9.

41. Merck. BMD efficacy and safety of odanacatib in postmenopausal women (0822-031). In: ClinicalTrials.gov [website on the Internet]. Bethseda, MD: US National Library of Medicine; 2008 [updated May 14, 2012]. Available from: clinicaltrials.gov/ct2/show/NCT00729183. NLM identifier: NCT00729183. 
42. Merck. A study to assess safety and efficacy of odanacatib (MK-0822) in men with osteoporosis (MK-0822-053 AM1). In: ClinicalTrials.gov [website on the Internet]. Bethseda, MD: US National Library of Medicine; 2010 [updated July 14, 2011]. Available from: clinicaltrials.gov/ ct2/show/NCT01120600. NLM identifier: NCT01120600.

43. Merck. A study of MK0822 in postmenopausal women with osteoporosis to assess fracture risk (MK-0822-018 AM4 EXT). In: ClinicalTrials.gov [website on the Internet]. Bethseda, MD: US National Library of Medicine; 2007 [updated March 16, 2012]. Available from: clinicaltrials.gov/ ct2/show/NCT00529373. NLM identifier: NCT00529373.

44. Horwitz MJ, et al Parathyroid hormone-related protein for the treatment of postmenopausal osteoporosis: defining the maximal tolerable dose. J Clin Endocrinol Metab 2010; 95(3): 1279-87.

45. Canalis E. New treatment modalities in osteoporosis. Endocr Pract 2010; 16(5): 855-63.

46. Takeuchi Y. Development of hPTHrP (1-36) as an anabolic therapeutic agent for osteoporosis. Clin Calcium 2011; 21(1): 28-32.

47. Nemeth EF. Calcimimetic and calcilytic drugs: just for parathyroid cells? Cell Calcium 2004; 35(3): 283-9.
48. Raje N, Vallet S. Sotatercept, a soluble activin receptor type 2A IgG-Fc fusion protein for the treatment of anemia and bone loss. Curr Opin Mol Ther 2010; 12(5): 586-97.

49. Canalis E. Update in new anabolic therapies for osteoporosis. JCEM 2010; 95(4): 1496-504.

50. Grisanti M, et al. Dkk-1 inhibition increases bone mineral density in rodents. J Bone Miner Res 2006; 21(1): S25 (Abstract).

51. Giles RH, van Es JH, Clevers H. Caught up in a Wnt storm: Wnt signaling in cancer. Biochim Biophys Acta 2003; 1653: $1-24$.

52. Canalis E, Centrella M, Burch W, McCarthy TL. Insulinlike growth factor I mediates selective anabolic effects of parathyroid hormone in bone cultures. J Clin Invest 1989; 83: $60-5$.

53. Ng KW. Potential role of odanacatib in the treatment of osteoporosis. Clinical Interventions in Aging 2012; 7: 235-47.

54. Lewiecki EM. New targets for intervention in the treatment of postmenopausal osteoporosis. Nat Rev Rheumatol 2011; 7(11): 631-8. 